\title{
3D modelling of branching in plants
}

\author{
J.B. Evers \\ Centre for Crop Systems Analysis, Wageningen University, Wageningen, the Netherlands \\ Email: jochem.evers@wur.nl
}

\begin{abstract}
Shoot branching is a key determinant of overall aboveground plant form. During plant development, the number of branches formed strongly influences the amount of light absorbed by the plant, and thus the plant's competitive strength in terms of light capture in relation to neighbouring plants. Branching is regulated by multiple internal factors which are modulated by different environmental signals.
\end{abstract}

A key environmental signal in the context of a plant population is a low red / far-red intensity ratio (R:FR) of the light reflected by neighbouring plants. For instance, low R:FR results in suppression of branching in favour of elongation growth, which is a key aspect of shade avoidance. Shade avoidance enables plants to anticipate future competition by preventing being shaded, rather than to react to prevailing shade conditions. Internally, branching is regulated by a finely tuned plant hormone network. The interactions within this network are modified by environmental cues such as R:FR which is perceived by specific photoreceptors. Combined, internal and external signals enable regulation of branch formation under the influence of environmental conditions.

The different aspects of branching control act at different levels of biological organization (organ, whole plant, plant community). These aspects can be integrated in one modelling approach, called functionalstructural plant modelling (FSPM), explicitly considering spatial 3D plant development. An FSP model typically contains detailed information at any moment in development of the plant on the number, size, location and orientation of all organs that make up the plant. In FSP models, physiological and physical processes occur within the plant (e.g. photosynthesis and transport of assimilates), and interaction with the environment occurs at the interface of organ and environment (e.g. light absorption by a leaf). Explicit simulation of absorption and scattering of light at the level of the plant organ is an important aspect of FSPM.

In combination with dedicated experiments, this modelling tool can be used to analyse the response of plants to (imminent) competition, simulate the competitive advantage of shade avoidance for plants of different architecture, and predict plant form in various light environments. To assess the effect of plant population density through R:FR signalling on tillering (branching) in spring wheat (Triticum aestivum L.), an FSPM study was conducted (Figure 1). A simple descriptive relationship was used to link R:FR as perceived by the plant to extension growth of tiller buds and probability of a bud to form a tiller. A further study included a complete sub-model of branching regulation, aiming at simulating branching as an emergent property in Arabidopsis (Arabidopsis thaliana) under the influence of R:FR. These and other studies show that FSPM is a promising tool to simulate aspects of plant development, such as branching, under the influence of environmental factors. In close combination with dedicated experiments, FSPM can shape our ideas of the mechanisms controlling plant development, can integrate existing knowledge on plant development, and can predict plant development in untested conditions.

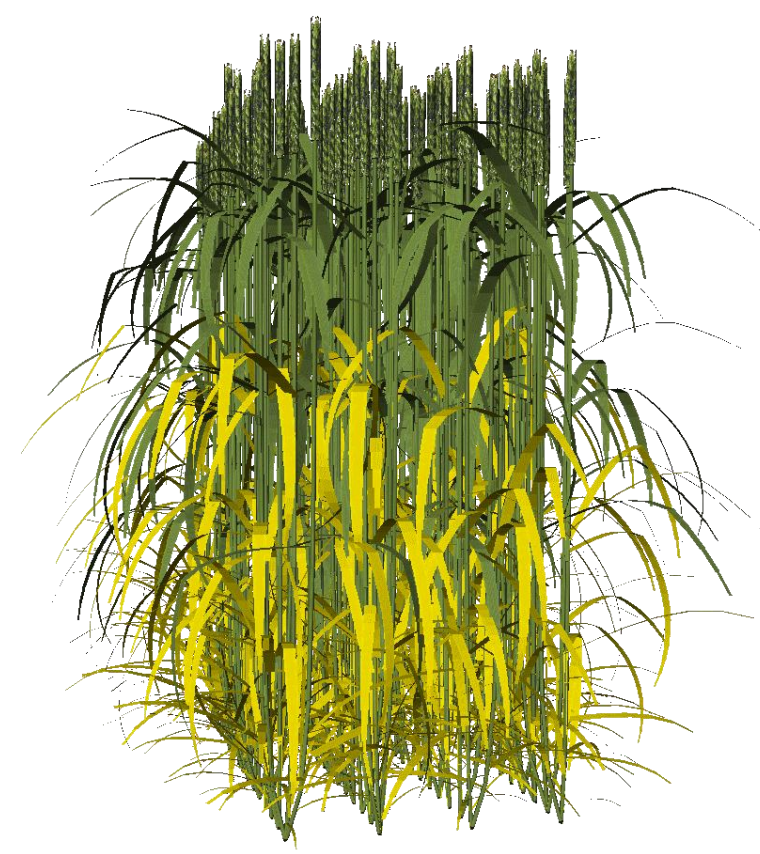

Figure 1. Simulated stand consisting of 4 rows of 15 wheat plants each, created using the FSPM methodology. Reprinted from Evers et al. (2011) with permission from Elsevier.

Keywords: $\quad$ functional-structural plant modelling (FSPM), plant hormone, light quality 


\section{INTRODUCTION}

Shoot branching is the production of side shoots from axillary buds on a plant stem. Currently research on the mechanisms involved in branching is booming with new insights in branching regulation published at a high rate (reviewed in Domagalska and Leyser, 2011). The branching behaviour observed in plants is the result of interacting processes occurring at different levels of biological organisation (i.e. cell, tissue, organ, whole plant, plant population). Therefore, in order to fully understand competitive relations between plants in natural and crop situations, we need to describe branching as an emergent behaviour emerging from interaction of processes both within and between plants. Integration of knowledge on mechanisms that regulate branching at different levels of biological organization is therefore important. Computational modelling has great potential as a tool to understand the complex interactions occurring in branching (Evers et al., 2011), as was also concluded earlier (Leyser, 2009; Crawford et al., 2010). The role of simulation modelling in branching research has been relatively small but significant (Dun et al., 2009b; Prusinkiewicz et al., 2009).

In this paper the significance of branching is outlined, and key factors involved in regulation of branching are reviewed and summarized in a conceptual model. The implementation of this conceptual model of regulation of branching in computational models using the so-called "functional-structural plant modeling" approach is discussed. Existing and forthcoming examples of simulation of branching are shown, and possibilities for future directions for the modelling approach in branching research are given.

\section{FUNCTIONAL-STRUCTURAL PLANT MODELLING (FSPM)}

Functional-structural plant modelling (abbreviated to FSPM hereafter), also termed virtual plant modelling, is a modelling approach to simulate plant growth and development. FSPM has been defined as a modelling technique "... explicitly describing the development over time of the 3D architecture or structure of plants as governed by physiological processes which, in turn, depend on environmental factors" (Vos et al., 2010). FSP models, or virtual plant models, have a rich history of variation in methods of implementation, in degree of complexity and biological realism, and in associated simulation platforms such as in L-Studio (Prusinkiewicz et al., 2000; Prusinkiewicz et al., 2007), GroIMP (Kniemeyer et al., 2007) and OpenAlea (Pradal et al., 2008), among other platforms. The common denominator of all FSPM approaches is the explicit inclusion of (aspects of) three-dimensional (3D) plant structure, architecture, shape, or morphology. This incorporation of plant architecture results from the general philosophy behind FSPM, which is that plant architecture is a concept central in plant growth and development. On the one hand, the genetic makeup of a plant, its physiological processes, and environmental factors (such as light, water, herbivory and competition for resources with other plants, etc.) determine development of plant architecture. On the other hand, plant architecture itself determines to what extent light can be intercepted, to what extent assimilates can be transported from a leaf to a growing tip, to what extent water and nutrients can be taken up from the soil by the roots, to what extent above- and below-ground signals can be perceived and broadcasted. Therefore, to better understand how plants with a specific genetic basis grow and develop in specific environmental conditions, the inclusion of plant architecture is essential. FSPM aims to provide the tools to analyse, simulate and predict (aspects of) plant growth and development, taking plant architecture into account.

Typically, an FSP model is defined and calibrated at the level of plant organs such as leaves, internodes (pieces of stem), root segments, etc. Most FSPM approaches use specific rules to define how organs develop and when new organs are created. By applying such rules repeatedly, growth and development of a plant can be simulated over time. The true power of FSPM emerges when the physiological and environmental processes that actually drive growth and development are added. For instance, by replacing rules that describe organ growth with routines for assimilate acquisition and allocation, and by adding calculation of light absorption and nutrient uptake, above- and below-ground growth can be simulated mechanistically, as an emergent property of the model (e.g. Dunbabin et al., 2004; Allen et al., 2005; Evers et al., 2010; Cieslak et al., 2011; Xu et al., 2011). Alternatively, an FSP model is not defined at the level of the plant organ but at lower levels such as the tissue or cell level; this approach has also been termed "computational morphodynamics" (Roeder et al., 2011). In such cases model output is not a whole plant or plant stand, but a tissue or organ such as a developing leaf (Bilsborough et al., 2011) or meristem (Barbier de Reuille et al., 2006).

\section{SHOOT BRANCHING}

Shoot branching is highly plastic plant trait: in conditions of low competition for resources a plant may produce many branches, whereas in competition a plant may remain branchless. Therefore branching is a 
key determinant of overall plant architecture. Plasticity in branching is a key element of shade avoidance (Franklin, 2008) which enables a plant to anticipate future competition for light by reducing resource investment in branching and investing more in height growth. However, as this strategy has a trade-off in the mechanical stability of the plant, a plant needs to be able to accurately assess the potential competitive capacity of the surrounding vegetation. That way it can regulate its branching patterns in anticipation of future conditions.

The process of branching can be decomposed into three processes: axillary bud formation, breaking of bud dormancy and subsequent branch growth. Bud formation is part of the plant developmental pattern. Depending on the species, one or more buds reside in the axil of a leaf as part of a phytomer (the combination of an internode, a leaf and an axillary bud), and the bud is formed when the leaf and the rest of the phytomer are formed. In determinate species, an environmental factor such as photoperiod (day length) can determine the total number of vegetative phytomers of a shoot and therefore the total number of buds that can grow into a branch (Yin and Kropff, 1996). Multiple internal factors that are modulated by environmental factors determine whether or not a bud will break and form a branch. These internal and environmental factors are part of a regulatory system with feedbacks at the level of the cell, the organ, the whole plant and even plant populations.

\subsection{Internal control of shoot branching}

When bud dormancy is broken, the bud initially needs assimilates to grow a branch and therefore is a strong sink for assimilates. If the assimilate source/sink ratio at plant level is high, branch growth can occur. However if the assimilate source/sink ratio is low (e.g. when the number of active buds is high, or the plant leaf area is relatively low), insufficient assimilates might be available and some active buds might not grow a branch. To prevent bud break at insufficient levels of assimilates to support branch growth, there is a coupling between sensing of assimilates (in the form of glucose) and bud break control; glucose has a direct effect on synthesis, transport and signalling of the plant hormone auxin (Mishra et al., 2009), a key player in hormonal branching control (see below). As the new branch develops, it gradually changes its role from sink to source for assimilates.

Bud break and subsequent branch growth are actively regulated by a hormone signalling network in which plant hormones auxin, cytokinin and strigolactone are the main hormonal factors (Leyser, 2009). Auxin reduces bud break through a phenomenon called apical dominance (Cline, 1991), which is effectuated by apical synthesis and subsequent basipetal transport of auxin. This basipetal transport is the result facilitated by auxin efflux carrier proteins (PINs) in the stem (Leyser, 2005). The suppression of bud break and branch growth by auxin is explained by the interplay of three signalling events.

- There is evidence that a positive feedback between auxin flux from cell to cell and the polar deposition of PIN proteins in cells represses branching (Prusinkiewicz et al., 2009; Balla et al., 2011). A reduction in apical auxin supply (e.g. through stem extension, decapitation, meristem death, or switch to flowering), reduces auxin flow in the main stem, which enhances and stabilizes auxin flow from the bud into the main auxin stream. The auxin efflux from the bud results in the formation of vascular connections (xylem and phloem) of the bud to the main vascular system. The new phloem connection allows assimilates, which are needed for branch growth, to be imported into the bud.

- Basipetal transport of auxin results in auxin signalling dependent down-regulation of synthesis of cytokinin, a potent promoter of branch outgrowth, in stem and root (Müller and Leyser, 2011). Dormant buds can be activated by cytokinin increase in the bud, due to a combination of an increase in local cytokinin synthesis and increased import via the vasculature (Dun et al., 2009a).

- Strigolactones are involved in repression of branching as well (Gomez-Roldan et al., 2008), for which there are two current hypotheses. (I) Auxin transported downwards in the stem stimulates synthesis of strigolactones in stem and roots (Hayward et al., 2009), which are transported upwards through the xylem (Kohlen et al., 2011). Strigolactones can then suppress bud break locally, inside the bud (Dun et al., 2009a). This hypothesis is supported by the result that strigolactone application directly to buds inhibit their outgrowth (Brewer et al., 2009). (II) Other evidence points to a more global role for strigolactones, setting the conditions for auxin transport in the shoot (Crawford et al., 2010). It was suggested that strigolactones control auxin transport (and thereby branching) by limiting PIN protein accumulation (Prusinkiewicz et al., 2009). Absence of strigolactone signalling would therefore result in higher concentrations of PIN protein and consequently increased auxin transport in the main stem, increased auxin export from buds into the main stem, and ultimately increased bud break. High auxin transport (Crawford et al., 2010) and branch numbers (Finlayson et al., 2010) are observed in Arabidopsis mutants deficient in strigolactone synthesis and/or response. 


\subsection{Environmental control of shoot branching}

Internal regulation of branching is modulated by external environmental factors. A high intensity of photosynthetically active radiation (PAR, ranging from $400 \mathrm{~nm}$ to $700 \mathrm{~nm}$ ) results in high assimilation rate and consequently a high source/sink ratio, which leads to (I) altered auxin transport and signalling activity (Mishra et al., 2009) leading to an increased number of vascularised buds, and (II) increased import of assimilates in these vascularised buds and therefore stimulation of branch growth. A low red / far-red intensity ratio (R:FR, 660:730 nm) is indicative of surrounding vegetation because red light is mainly absorbed by green plant parts, and far-red light is mainly scattered. Low R:FR is one of the signals that elicit shade avoidance responses in plants, leading to suppression of bud break (Casal, 1988; Finlayson et al., 2010). Plants have phytochrome photoreceptors in most plant tissues in order to perceive R:FR. Phytochromes exist in two forms $\left(\mathrm{P}_{\mathrm{r}}\right.$ and the biologically active $\left.\mathrm{P}_{\mathrm{fr}}\right)$, which are converted into each other upon absorption of red or far-red light, respectively. Thus, the balance between the two forms, called the photoequilibrium $\left(\mathrm{P}_{\mathrm{fr}} / \mathrm{P}_{\text {total }}\right)$, is a reflection of the R:FR locally perceived (Smith, 2000). $\mathrm{P}_{\mathrm{r}}$ suppresses local auxin synthesis, transport and responsiveness (e.g. Keuskamp et al., 2010). Low R:FR thus directly affects the hormone signalling network related to bud break. A growing branch can, in turn, reduce PAR and R:FR in the surrounding environment, reducing the branching potential of other buds.

Soil nitrogen $(\mathrm{N})$ limitation can directly suppress branch growth independent of any direct hormonal control (McIntyre, 2001). Soil phosphorus (P) limitation results in decreased branching (Kohlen et al., 2011), and also in increased secretion of strigolactones into the rhizosphere, to stimulate interaction with symbiotic mycorrhizal fungi, that increases the plant's P uptake (López-Ráez and Bouwmeester, 2008). Together, these two effects of low soil P strongly suggest a role of strigolactones in branching control, as messengers of soil $\mathrm{P}$ status, suppressing branch growth under P-limited conditions. Additionally, $\mathrm{N}$ and $\mathrm{P}$ also modulate levels cytokinin, which in turn regulate nutrient acquisition (e.g. Kiba et al., 2011), potentially affecting branching directly or indirectly.

Active manipulation of plant structure, such as through herbivory, mowing, pruning and harvesting, also affects branching, in multiple ways. New grass shoots grow after defoliation by grazing or mowing (Gautier et al., 1999), and new branches form after pruning other branches or after removing even larger parts of the plant structure such as in orchard trees (Lauri et al., 2009). The effects of manipulation of plant structure on branching can be the combined result of (i) the removal of auxin source(s) and the associated effects on the entire hormonal regulatory network; (ii) changes in the light intensity and spectral composition within the plant structure and the associated effects on phytochrome action and assimilate supply, respectively, and (iii) the removal of sources or sinks for assimilates and the associated changes in the source/sink ratios of the plant. This makes active manipulation of plant structure perhaps the most outstanding example to illustrate the complexity of interacting factors affecting branching at several levels of biological organization.

\section{SIMULATING SHOOT BRANCHING USING FSPM}

To be able to support research on branching, the FSPM approach has several distinctive properties to offer. (I) Simulation of plant topology, i.e. the (2D) structure of the network of interconnected organs. This allows simulation of transport of compounds such as assimilates or hormones through the plant from source to sink organ, taking into account the number of nodes to travel and the number of ramifications to encounter. (II) Simulation of (3D) geometry of the plant and its organs. A detailed geometric description of plant architecture enables simulation of light interception from any direction on leaves and other organs. This enables calculation of local and whole-plant light absorption, which is vital for simulation of photosynthesis and photomorphogenetic processes affecting branching. (III) Integration of several levels of biological organization. For example, in FSPM physiological processes can be defined at the level of, for instance, the

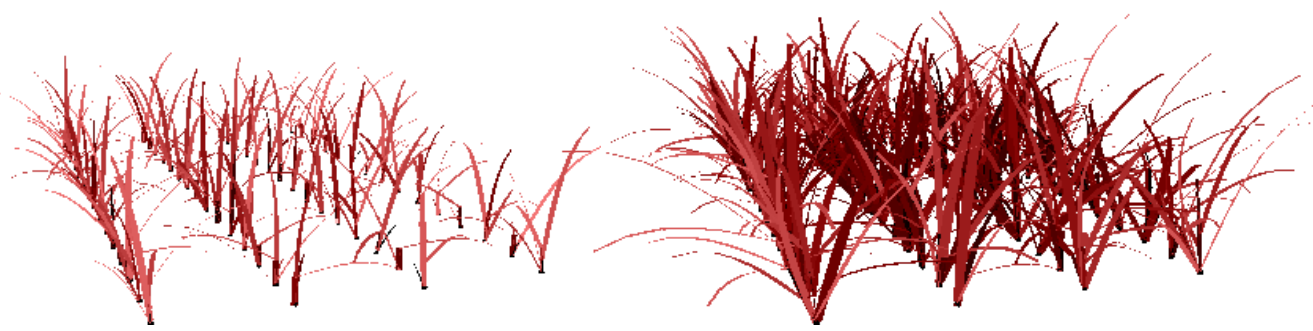

Figure 2. Two stages of early development of stands consisting of 4 rows of 15 wheat plants each, created using the FSPM methodology. The colour of the leaves represents the R:FR ratio perceived by the leaf. Right figure reprinted from Evers et al. (2011) with permission from Elsevier. 
leaf, the axillary bud or the shoot apical meristem. Output can be generated at the level of the whole plant and plant communities. Subsequently, processes at the level of whole plants or communities such as light scattering affect low-level processes such as phytochrome action and associated photomorphogenesis.

These aspects make FSPM a methodology highly suitable to simulate branching. Several attempts using FSPM to integrate aspects of branching have been performed in the recent past. Prusinkiewicz et al. (2009) constructed a model of bud break based on the assumption that buds break only when they can export locally synthesized auxin into the auxin flow of the parent shoot. This model suggested that dormant buds do not grow out due to the positive feedback between polarization of auxin transport and auxin flux in the mother shoot. The model faithfully reproduced branching patterns such as acropetal branching, top branching upon removal of the apical meristem, and extensive branching in strigolactone mutants. The approach was based on a schematic representation of plant architecture, and did not include any external influence on bud break. Another FSPM approach simulating branching did include realistic plant architecture and environmental influence, but lacked a proper physiological connection between environment and branching (Evers et al., 2007). In this study branching in wheat was made dependent on a direct relationship between R:FR as perceived by the plant and bud growth (figure 2). Branching patterns in relation to plant population density and associated light environment were reproduced realistically, depending on the type of functional relationship between R:FR and bud growth chosen.

Recently, an attempt was made to integrate hormone signalling, R:FR signalling, realistic plant architecture, plant manipulation and their feedback effects in one FSP model of Arabidopsis development (Evers and van der Krol, 2010) (Figure 3). In this work, an architectural model of Arabidopsis development was constructed using the modelling platform GroIMP (Hemmerling et al., 2008), and calibrated using published data (Mündermann et al., 2005). Organs were provided with capabilities to synthesize, import and export hormones, to scatter red and far-red light differentially, to sense R:FR of the perceived light and adjust auxin synthesis accordingly. Bud break and branch growth were made dependent on auxin export from the bud and cytokinin level within the bud, respectively. Simulated plants produced varying numbers of branches depending on R:FR of the incoming light, simulated deficiencies in hormone signalling (mutations), and simulated removal of the growing apex. More details on this work will be provided in forthcoming communications.

\section{CONCLUSIONS AND RECOMMENDATIONS}

Functional-structural plant modelling (FSPM) is a promising tool to simulate aspects of plant development, such as branching, under the influence of environmental factors. FSPM can cover a range of levels of biological organization, making it suitable to simulate whole plant and plant population responses as emergent behaviour of organ and sub-organ level processes. In close combination with dedicated experiments, FSPM can help us to integrate knowledge on plant development, to test and define new hypotheses on the mechanisms governing plant development, and to predict plant development in a range of conditions.
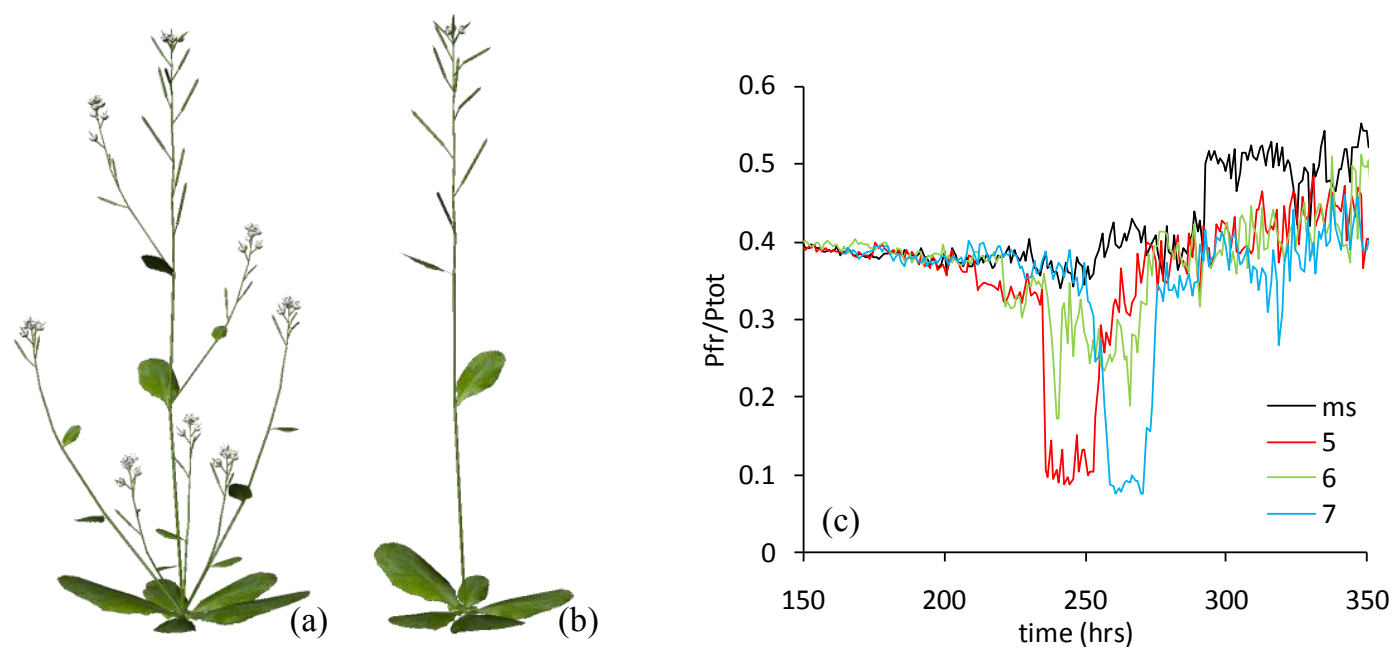

Figure 3. Arabidopsis plants simulated (a) at $\mathrm{R}: \mathrm{FR}=1.2$ (normal daylight), (b) $\mathrm{R}: \mathrm{FR}=0.01$ (high density canopy shade). (c) $\mathrm{P}_{\mathrm{fr}} / \mathrm{P}_{\text {tot }}$ at the main stem apex (ms) and axillary meristems located in the axils of rosette leaves 5, 6 and 7; simulated at ambient R:FR of 1.2. From Evers and Van der Krol (2010). 


\section{ACKNOWLEDGEMENTS}

The input of A.R. van der Krol and J. Vos is gratefully acknowledged. Financial support was provided by the Netherlands Organization for Scientific Research (NWO Veni grant no. 86308012).

\section{REFERENCES}

Allen MT, Prusinkiewicz P, DeJong TM. 2005. "Using L-systems for modeling source-sink interactions, architecture and physiology of growing trees: the L-PEACH model." New Phytologist 166(3): 869880.

Balla J, Kalousek P, Reinöhl V, Friml J, Procházka S. 2011. "Competitive canalization of PIN-dependent auxin flow from axillary buds controls pea bud outgrowth." the Plant Journal 65(4): 571-577.

Barbier de Reuille P, Bohn-Courseau I, Ljung K, Morin H, Carraro N, Godin C, Traas J. 2006. "Computer simulations reveal properties of the cell-cell signaling network at the shoot apex in Arabidopsis." Proceedings of the National Academy of Sciences USA 103(5): 1627-1632.

Bilsborough GD, Runions A, Barkoulas M, Jenkins HW, Hasson A, Galinha C, Laufs P, Hay A, Prusinkiewicz P, Tsiantis M. 2011. "Model for the regulation of Arabidopsis thaliana leaf margin development." Proceedings of the National Academy of Sciences USA 108(8): 3424-3429.

Brewer PB, Dun EA, Ferguson BJ, Rameau C, Beveridge CA. 2009. "Strigolactone acts downstream of auxin to regulate bud outgrowth in pea and arabidopsis." Plant Physiology 150(1): 482-493.

Casal JJ. 1988. "Light quality effects on the appearance of tillers of different order in wheat (Triticum aestivum)." Annals of Applied Biology 112: 167-173.

Cieslak M, Seleznyova AN, Hanan J. 2011. "A functional-structural kiwifruit vine model integrating architecture, carbon dynamics and effects of the environment." Annals of Botany 107(5): 747-764.

Cline MG. 1991. "Apical dominance." Botanical Review 57(4): 318-358.

Crawford S, Shinohara N, Sieberer T, Williamson L, George G, Hepworth J, Muller D, Domagalska MA, Leyser O. 2010. "Strigolactones enhance competition between shoot branches by dampening auxin transport." Development 137(17): 2905-2913.

Domagalska MA, Leyser O. 2011. "Signal integration in the control of shoot branching." Nature Reviews Molecular Cell Biology 12(4): 211-221.

Dun EA, Brewer PB, Beveridge CA. 2009a. "Strigolactones: discovery of the elusive shoot branching hormone." Trends in Plant Science 14(7): 364-372.

Dun EA, Hanan J, Beveridge CA. 2009b. "Computational modeling and molecular physiology experiments reveal new insights into shoot branching in pea." Plant Cell 21(11): 3459-3472.

Dunbabin V, Rengel Z, Diggle AJ. 2004. "Simulating form and function of root systems: Efficiency of nitrate uptake is dependent on root system architecture and the spatial and temporal variability of nitrate supply." Functional Ecology 18(2): 204-211.

Evers JB, Vos J, Chelle M, Andrieu B, Fournier C, Struik PC. 2007. "Simulating the effects of localized red:far-red ratio on tillering in spring wheat (Triticum aestivum) using a three-dimensional virtual plant model." New Phytologist 176(2): 325-336.

Evers JB, van der Krol AR. 2010. "Hormonal regulation of branching modulated by light quality". 6th International Workshop on Functional-Structural Plant Models. Davis, USA, 149-151.

Evers JB, Vos J, Yin X, Romero P, van der Putten PEL, Struik PC. 2010. "Simulation of wheat growth and development based on organ-level photosynthesis and assimilate allocation." Journal of Experimental Botany 61(8): 2203-2216.

Evers JB, van der Krol AR, Vos J, Struik PC. 2011. "Understanding shoot branching by modelling form and function." Trends in Plant Science 16(9): doi: 10.1016/j.tplants.2011.05.004.

Finlayson SA, Krishnareddy SR, Kebrom TH, Casal JJ. 2010. "Phytochrome regulation of branching in arabidopsis." Plant Physiology 152(4): 1914-1927.

Franklin KA. 2008. "Shade avoidance." New Phytologist 179(4): 930-944.

Gautier H, Varlet-Grancher C, Hazard L. 1999. "Tillering responses to the light environment and to defoliation in populations of perennial ryegrass (Lolium perenne L.) selected for contrasting leaf length." Annals of Botany 83: 423-429.

Gomez-Roldan V, Fermas S, Brewer PB, Puech-Pagès V, Dun EA, Pillot JP, Letisse F, Matusova R, Danoun S, Portais JC, Bouwmeester H, Bécard G, Beveridge CA, Rameau C, Rochange SF. 2008. "Strigolactone inhibition of shoot branching." Nature 455(7210): 189-194.

Hayward A, Stirnberg P, Beveridge C, Leyser O. 2009. "Interactions between auxin and strigolactone in shoot branching control." Plant Physiology 151(1): 400-412.

Hemmerling R, Kniemeyer O, Lanwert D, Kurth W, Buck-Sorlin GH. 2008. "The rule-based language XL and the modelling environment GroIMP illustrated with simulated tree competition." Functional Plant Biology 35(10): 739-750. 
Keuskamp DH, Pollmann S, Voesenek LACJ, Peeters AJM, Pierik R. 2010. "Auxin transport through PINFORMED 3 (PIN3) controls shade avoidance and fitness during competition." Proceedings of the National Academy of Sciences USA 107(52): 22740-22744.

Kiba T, Kudo T, Kojima M, Sakakibara H. 2011. "Hormonal control of nitrogen acquisition: roles of auxin, abscisic acid, and cytokinin." Journal of Experimental Botany 62(4): 1399-1409.

Kniemeyer O, Buck-Sorlin GH, Kurth W. 2007. "GroIMP as a platform for functional-structural modelling of plants." In: Vos J, Marcelis LFM, de Visser PHB, Struik PC and Evers JB, eds. FunctionalStructural Plant Modelling in Crop Production. Dordrecht, the Netherlands: Springer, 43-52.

Kohlen W, Charnikhova T, Liu Q, Bours R, Domagalska MA, Beguerie S, Verstappen F, Leyser O, Bouwmeester HJ, Ruyter-spira C. 2011. "Strigolactones are transported through the xylem and play a key role in shoot architectural response to phosphate deficiency in non-AM host Arabidopsis thaliana." Plant Physiology 155: 974-987.

Lauri PA, Costes E, Regnard JL, Brun L, Simon S, Monney P, Sinoquet H. 2009. "Does knowledge on fruit tree architecture and its implications for orchard management improve horticultural sustainability? An overview of recent advances in the apple." Acta Horticulturae 817: 243-250.

Leyser O. 2005. "Auxin distribution and plant pattern formation: how many angels can dance on the point of PIN?" Cell 121(6): 819-822.

Leyser O. 2009. "The control of shoot branching: an example of plant information processing." Plant, Cell \& Environment 32(6): 694-703.

López-Ráez JA, Bouwmeester HJ. 2008. "Fine-tuning regulation of strigolactone biosynthesis under phosphate starvation." Plant Signaling and Behavior 3(11): 963-965.

McIntyre GI. 2001. "Control of plant development by limiting factors: a nutritional perspective." Physiologia Plantarum 113(2): 165-175.

Mishra BS, Singh M, Aggrawal P, Laxmi A. 2009. "Glucose and auxin signaling interaction in controlling Arabidopsis thaliana seedlings root growth and development." PLoS ONE 4(2): e4502.

Müller D, Leyser O. 2011. "Auxin, cytokinin and the control of shoot branching." Annals of Botany 107(7): 1203-1212.

Mündermann L, Erasmus Y, Lane B, Coen E, Prusinkiewicz P. 2005. "Quantitative modeling of Arabidopsis development." Plant Physiology 139(2): 960-968.

Pradal C, Dufour-Kowalski S, Boudon F, Fournier C, Godin C. 2008. "OpenAlea: A visual programming and component-based software platform for plant modelling." Functional Plant Biology 35(10): 751-760.

Prusinkiewicz P, Karwowski R, Mĕch R, Hanan JS. 2000. "L-studio/CPFG: a software system for modeling plants." In: Nagl M, Schürr A and Münch M, eds. Applications of Graph Transformations with Industrial Relevance. Lecture notes in Computer Science 1779. Berlin, Germany: Springer, $457-$ 464.

Prusinkiewicz P, Karwowski R, Lane B. 2007. "Modelling architecture of crop plants using L-systems." In: Vos J, Marcelis LFM, de Visser PHB, Struik PC and Evers JB, eds. Functional-Structural Plant Modelling in Crop Production. Dordrecht, the Netherlands: Springer, 27-42.

Prusinkiewicz P, Crawford S, Smith RS, Ljung K, Bennett T, Ongaro V, Leyser O. 2009. "Control of bud activation by an auxin transport switch." Proceedings of the National Academy of Sciences USA 106(41): 17431-17436.

Roeder AHK, Tarr PT, Tobin C, Zhang X, Chickarmane V, Cunha A, Meyerowitz EM. 2011. "Computational morphodynamics of plants: Integrating development over space and time." Nature Reviews Molecular Cell Biology 12(4): 265-273.

Smith H. 2000. "Phytochromes and light signal perception by plants - an emerging synthesis." Nature 407: 585-591.

Vos J, Evers JB, Buck-Sorlin GH, Andrieu B, Chelle M, de Visser PHB. 2010. "Functional-structural plant modelling: a new versatile tool in crop science." Journal of Experimental Botany 61(8): 21022115.

Xu L, Henke M, Zhu J, Kurth W, Buck-Sorlin G. 2011. "A functional-structural model of rice linking quantitative genetic information with morphological development and physiological processes." Annals of Botany 107(5): 817-828.

Yin X, Kropff MJ. 1996. "Use of the Beta function to quantify effects of photoperiod on flowering and leaf number in rice." Agricultural and Forest Meteorology 81(3-4): 217-228. 